A 73-year-old man was admitted with cholangitis. He had undergone a Billroth II gastrectomy 20 years before. His liver function tests showed an obstructive pattern of abnormalities and ultrasound revealed a dilated common bile duct with stones at the distal end. Emergency endoscopic retrograde cholangiopancreatography (ERCP) was commenced but the papilla could not be identified with the duodenoscope in the afferent loop. An end-viewing gastroscope was used to repeat the examination, and the papilla was found to be situated very close to the stump of the afferent loop and the opening was facing backwards, which made cannulation of the bile duct impossible (Figure 1a). A transparent cap (Olympus Distal Attachments MH-593; Olympus Tokyo, Japan) was then attached to the gastroscope and the examination was repeated. The side wall of the papilla was pushed with the cap and the opening was turned to face the scope (Figure $\mathbf{1 b}$ ). The bile duct was then cannulated and a stent was inserted for drainage (Figure 2). The cholangiogram showed three $1-\mathrm{cm}$ stones at the distal end of the common bile duct.

At a second endoscopy session it was not possible to perform a sphincterotomy because there was no room for manipulation of the scope. Instead, a balloon sphincteroplasty was performed and the papilla was

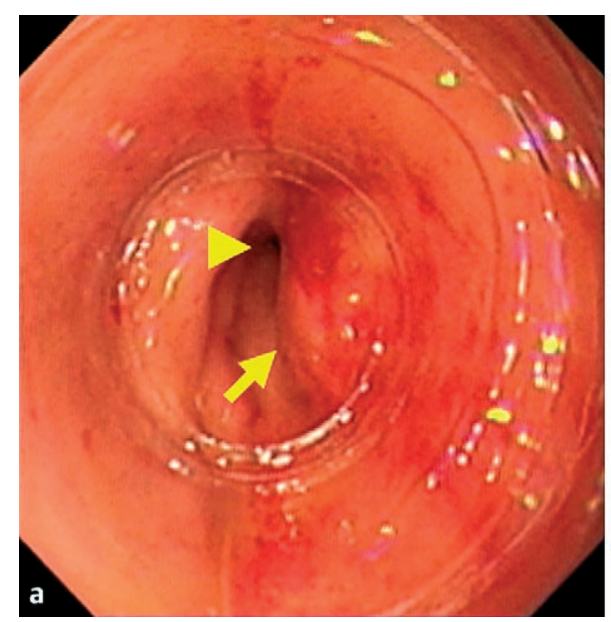

Figure 1 Endoscopic view of the papilla: a before pushing by the cap, showing the papilla (arrow) close to the stump of the afferent

\title{
Cap-Assisted Endoscopic Retrograde Cholangiopancreatography in a Patient with a Billroth II Gastrectomy
}

dilated up to $10 \mathrm{~mm}$. The bile duct stones were removed with a wire-guided basket and the patient recovered uneventfully.

Cap-assisted endoscopy has been widely used in mucosectomy procedures for the treatment of early gastric cancer [1] and can also facilitate the detection and visualization of lesions that are situated in "blind areas" of the gastrointestinal tract $[2,3]$. The technique can also be used to manipulate the position of a lesion or of an anatomical structure, such as the papilla in this case, in order to facilitate endoscopic therapy. The cap can stabilize the endoscope tip while leaving enough room for endoscopic therapy [2]. Whether or not the routine use of a cap in assisting ERCP in patients with a history of Billroth II gastrectomy improves the cannulation rate requires further study. Although sphincterotomy using an end-viewing endoscope can be difficult, the use of balloon papillary dilation was shown to be advantageous in this situation, in achieving the same ductal clearance rate while decreasing the risk of post-sphincterotomy bleeding [4].

\section{Y. T. Lee}

Department of Medicine and

Therapeutics, Prince of Wales Hospital, Chinese University of Hong Kong, Shatin, Hong Kong, China.

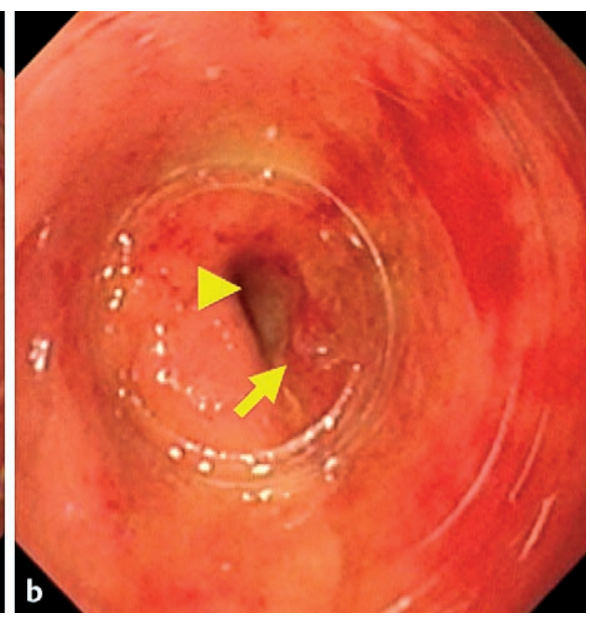

loop (arrowhead) and facing backwards; and b after pushing by the cap, showing the papilla displaced en face to the endoscope.

\section{References}

${ }^{1}$ Matsuzaki K, Nagao S, Kawaguchi Aet al. Newly designed soft prelooped cap for endoscopic mucosal resection of gastric lesions. Gastrointest Endosc 2003; 57: 242-246

${ }^{2}$ Yap CK, Ng HS. Cap-fitted gastroscopy improves visualization and targeting of lesions. Gastrointest Endosc 2001; 53: 93-95

${ }^{3}$ Matsushita M, Hajiro K, Okazaki Ket al. Efficacy of total colonoscopy with a transparent cap in comparison with colonoscopy without a cap. Endoscopy 1998; 30: 444-447

${ }^{4}$ Shah SK, Mutignani M, Costamagna G. Therapeutic biliary endoscopy. Endoscopy 2002; 34: $43-53$

\section{Corresponding Author}

\section{Y. T. Lee, MRCP, MD}

Department of Medicine and Therapeutics

The Chinese University of Hong Kong

Prince of Wales Hospital

Shatin N.T.

Hong Kong SAR

China

Fax: $\quad$ +852-2637-5396

E-mail: leeytong@cuhk.edu.hk

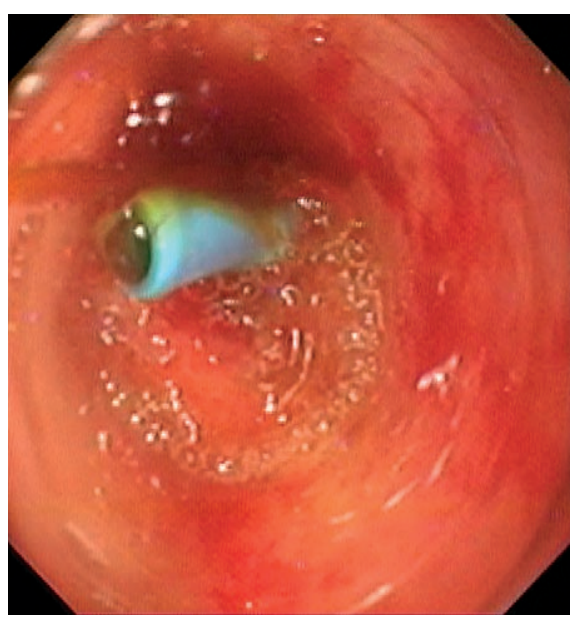

Figure 2 A biliary stent was inserted after successful cannulation of the bile duct. 